PROCEEDINGS OF THE

AMERICAN MATHEMATICAL SOCIETY

Volume 130, Number 3, Pages 863-874

S 0002-9939(01)06082-8

Article electronically published on June 20, 2001

\title{
SOME APPLICATIONS OF THE ADAMS-KECHRIS TECHNIQUE
}

\author{
SU GAO \\ (Communicated by Carl G. Jockusch, Jr.)
}

\begin{abstract}
We analyze the technique used by Adams and Kechris (2000) to obtain their results about Borel reducibility of countable Borel equivalence relations. Using this technique, we show that every $\boldsymbol{\Sigma}_{1}^{1}$ equivalence relation is Borel reducible to the Borel bi-reducibility of countable Borel equivalence relations. We also apply the technique to two other classes of essentially uncountable Borel equivalence relations and derive analogous results for the classification problem of Borel automorphisms.
\end{abstract}

\section{INTRODUCTION}

For equivalence relations $E$ and $F$ on standard Borel spaces $X$ and $Y$ respectively, we say that $E$ is Borel reducible to $F$ and write $E \leq_{B} F$ if there is a Borel function $f: X \rightarrow Y$ such that, for every $x, y \in X, x E y$ iff $f(x) F f(y)$. Furthermore, $E$ is said to be Borel bi-reducible to $F$ and denoted $E \sim_{B} F$ if both $E \leq_{B} F$ and $F \leq_{B} E$. Let $\mathcal{E}$ be the class of all Borel equivalence relations and let $\mathcal{E}^{\text {ctbl }}$ be the class of all countable Borel equivalence relations, i.e., those relations in $\mathcal{E}$ whose equivalence classes are countable. The structures $\left(\mathcal{E}, \leq_{B}\right)$ and $\left(\mathcal{E}^{\text {ctbl }}, \leq_{B}\right)$ have been investigated quite extensively in recent years. In [1] Adams and Kechris developed a powerful technique to deal with countable Borel equivalence relations and obtained a number of important results. A sample of these results is the following embedding theorem:

Theorem 1 (Adams-Kechris). The structure of Borel sets with inclusion can be embedded into $\left(\mathcal{E}^{\mathrm{ctbl}}, \leq_{B}\right)$.

Previously Louveau and Veclickovic had shown that the structure $\left(\mathcal{P}(\omega), \subseteq_{*}\right)$ can be embedded into $\left(\mathcal{E}, \leq_{B}\right)$, where $\mathcal{P}(\omega)$ is the power set of $\omega$ and $\subseteq_{*}$ denotes inclusion modulo finite sets $([10])$; whereas for $\left(\mathcal{E}^{\text {ctbl }}, \leq_{B}\right)$ there had only been an observation that it contains an increasing chain with four elements.

Let $\sim_{B}$ denote the equivalence relation of Borel bi-reducibility on $\mathcal{E}$ and let $\sim_{B}^{\text {ctbl }}$ be the restriction of $\sim_{B}$ on $\mathcal{E}^{\mathrm{ctbl}}$. The embedding in Theorem 1 is in fact effective so as to give the following corollary.

Theorem 2 (Adams-Kechris). The equality of Borel sets is Borel reducible to $\sim_{B}^{\text {ctbl }}$. Consequently, every Borel equivalence relation is Borel reducible to $\sim_{B}^{\text {ctbl }}$.

Received by the editors February 10, 2000 and, in revised form, August 13, 2000 and August 23, 2000.

1991 Mathematics Subject Classification. Primary 03E15.

Key words and phrases. Borel equivalence relations, Borel (bi-)reducibility.

(C)2001 American Mathematical Society 
With appropriate codings, the equality of Borel sets becomes a $\boldsymbol{\Pi}_{1}^{1}$ equivalence relation and $\sim_{B}^{\text {ctbl }}$ can be seen to be $\boldsymbol{\Sigma}_{2}^{1}$. Adams and Kechris also showed that $\sim_{B}^{\text {ctbl }}$ is $\boldsymbol{\Sigma}_{2}^{1}$-complete.

In addition, they also considered the weaker reducibility by $\sigma\left(\boldsymbol{\Sigma}_{1}^{1}\right)$-measurable functions. Here $\sigma\left(\boldsymbol{\Sigma}_{1}^{1}\right)$ denotes the smallest $\sigma$-algebra containing all $\boldsymbol{\Sigma}_{1}^{1}$ sets. In the sequel we replace the subscript $B$ by $S$ when we deal with $\sigma\left(\boldsymbol{\Sigma}_{1}^{1}\right)$-measurable reductions instead of Borel ones. In [1] it was also proved that $\sim_{S}^{\text {ctbl }}$ is $\Pi_{2}^{1}$-hard and that there are $E, F \in \mathcal{E}^{\mathrm{ctbl}}$ such that $E \leq_{S} F$ but $E \underline{\leq}_{B} F$.

There were a number of new ideas involved in proving these important results, but as we shall see in the next section, there is a very general and powerful technique which lies in the core of several arguments. By isolating this technique we are able to improve some of the results in [1] and also apply it to various other situations. Specifically, we shall use the technique to prove the following theorems.

Theorem 3. Every $\Sigma_{1}^{1}$ equivalence relation is Borel reducible to $\sim_{B}^{\text {ctbl }}$.

Theorem 4. Every $\boldsymbol{\Sigma}_{1}^{1}$ or $\boldsymbol{\Pi}_{1}^{1}$ equivalence relation is Borel reducible to $\sim_{S}^{\text {ctbl }}$.

In order to state the other results we need some more notation. Given equivalence relations $E$ and $F$ with $E<_{B} F$, we let

$$
[E, F]=\left\{R \in \mathcal{E} \mid E \leq_{B} R \leq_{B} F\right\}
$$

and call it the interval between $E$ and $F$. Recall that there is a universal countable Borel equivalence relation $E_{\infty}$, i.e., $E_{\infty}$ is a largest element in the structure $\left(\mathcal{E}^{\mathrm{ctbl}}, \leq_{B}\right)$. Also the classical Glimm-Effros dichotomy theorem of HarringtonKechris-Louveau implies that there is a smallest non-smooth element in $\left(\mathcal{E}, \leq_{B}\right)$, namely $E_{0}$, the Vitali equivalence relation (cf., e.g., 2]). Putting all the notation together, Theorem 1 can be restated in a slightly weaker form as: the structure of Borel sets with inclusion can be embedded into $\left(\left[E_{0}, E_{\infty}\right], \leq_{B}\right)$. Here the interval $\left[E_{0}, E_{\infty}\right]$ is the class of all Borel equivalence relations which are essentially countable yet non-smooth.

We would like to apply the Adams-Kechris technique to essentially uncountable Borel equivalence relations, i.e., those that are not essentially countable. For this we consider the following operation on equivalence relations. Given an equivalence relation $E$ on a standard Borel space $X$, we denote by $E^{\omega}$ the equivalence relation on $X^{\omega}$ defined by

$$
\left(x_{n}\right) E^{\omega}\left(y_{n}\right) \Leftrightarrow \forall n\left(x_{n} E y_{n}\right) .
$$

It is well known that $E_{0}^{\omega}$ is not essentially countable (in fact, not essentially $F_{\sigma}$ ). Therefore all the equivalence relations in the interval $\left[E_{0}^{\omega}, E_{\infty}^{\omega}\right]$ are not essentially countable. However, the Adams-Kechris technique applies.

Theorem 5. The structure of Borel sets with inclusion can be embedded into $\left(\left[E_{0}^{\omega}, E_{\infty}^{\omega}\right], \leq_{B}\right)$.

Other results similar to Adams-Kechris' can be similarly obtained.

Theorem 6. The following facts hold for the interval $\mathcal{I}=\left[E_{0}^{\omega}, E_{\infty}^{\omega}\right]$ :

(a) Every $\Sigma_{1}^{1}$ equivalence relation is Borel reducible to $\sim_{B}^{\mathcal{I}}$, the restriction of $\sim_{B}$ to $\mathcal{I}$.

(b) Every $\boldsymbol{\Sigma}_{1}^{1}$ or $\boldsymbol{\Pi}_{1}^{1}$ equivalence relation is Borel reducible to $\sim_{S}^{\mathcal{I}}$.

(c) $\sim_{B}^{\mathcal{I}}$ is $\boldsymbol{\Sigma}_{2}^{1}$-complete. 
(d) $\sim_{S}^{\mathcal{I}}$ is $\boldsymbol{\Pi}_{2}^{1}$-hard.

(e) There are $E, F \in \mathcal{I}$ such that $E \leq_{S} F$ but $E \not{ }_{B} F$.

A map $f$ from a standard Borel space $X$ to the set $\left[2^{\mathbb{N}}\right]^{\omega}$ of countable sets of reals is said to be Borel if there is a Borel map $g: X \rightarrow\left(2^{\mathbb{N}}\right)^{\omega}$ such that, for any $x \in X$,

$$
f(x)=\{g(x)(n) \mid n \in \omega\} .
$$

Note that $E_{\infty}^{\omega}$ is, in a natural sense in accordance with the above definition, Borel reducible to the equality relation of countable sets of reals. In fact, the countable set of reals assigned to the element $\left(x_{n}\right) \in\left(2^{\mathbb{N}}\right)^{\omega}$ as a complete invariant can be $\left\{\langle n, z\rangle \mid z E_{\infty} x_{n}, n \in \omega\right\}$. Moreover, it is easy to code countable sets of reals up to identity by countable structures (in some countable language) up to isomorphism (cf., e.g. section 2 of 8 for a thorough treatment of these remarks). Therefore, overall, one can assign countable structures as complete invariants for the equivalence classes of $E_{\infty}^{\omega}$.

In the next application we step further away to consider the class

$$
\mathcal{E}^{\text {turb }}=\left\{R \in \mathcal{E} \mid R \not_{B} \text { the isomorphism of countable structures }\right\} .
$$

The superscript turb comes from Hjorth's theory of turbulence for this class of equivalence relations (cf. 7]). It follows that none of the equivalence relations we considered above belongs to $\mathcal{E}^{\text {turb }}$. Nevertheless, the Adams-Kechris technique applies to $\mathcal{E}^{\text {turb }}$ as well. We will show a complete analogue of the results again.

Theorem 7. The following hold for $\mathcal{E}^{\text {turb }}$ :

(a) The structure of Borel sets with inclusion can be embedded into $\left(\mathcal{E}^{\text {turb }}, \leq_{B}\right)$.

(b) Every $\boldsymbol{\Sigma}_{1}^{1}$ equivalence relation is Borel reducible to $\sim_{B}^{\text {turb }}$.

(c) Every $\boldsymbol{\Sigma}_{1}^{1}$ or $\boldsymbol{\Pi}_{1}^{1}$ equivalence relation is Borel reducible to $\sim_{S}^{\text {turb }}$.

(d) $\sim_{B}^{\text {turb }}$ is $\boldsymbol{\Sigma}_{2}^{1}$-complete.

(e) $\sim_{S}^{\text {turb }}$ is $\Pi_{2}^{1}$-hard.

(f) There are $E, F \in \mathcal{E}^{\text {turb }}$ such that $E \leq_{S} F$ but $E \not_{B} F$.

Finally, the Adams-Kechris technique can even be applied to study objects other than equivalence relations. Following [3] we can consider Borel embeddings and Borel isomorphisms of Borel automorphisms. The complete analogy is preserved and we obtain

Theorem 8. Every $\boldsymbol{\Sigma}_{1}^{1}$ equivalence relation is Borel reducible to the Borel isomorphism of Borel automorphisms. Every $\boldsymbol{\Sigma}_{1}^{1}$ or $\boldsymbol{\Pi}_{1}^{1}$ equivalence relation is Borel reducible to the $\sigma\left(\boldsymbol{\Sigma}_{1}^{1}\right)$-measurable isomorphism of Borel automorphisms.

The paper is organized as follows. In section 2 we prove Theorems 3 and 4 and extract the generalities of the Adams-Kechris technique. Section 3 deals with Theorems 5 and 6 . Section 4 deals with Theorem 7 . In the last section we remark on Theorem 8 and discuss some further questions. The author is grateful to A. S. Kechris and G. Hjorth for inspiring conversations.

\section{Elements of the technique}

In this section we prove Theorems 3 and 4 and extract from the proofs the basic elements of the technique used by Adams and Kechris in [1. The proofs here will be mostly self-contained except for the following fundamental theorem in [1] resulting from a deep analysis in ergodic theory. 
Theorem 9 (Adams-Kechris). There is a map $x \mapsto E_{x}$ assigning to each $x \in 2^{\mathbb{N}}$ a countable Borel equivalence relation $E_{x}$ on $2^{\mathbb{N}}$ such that

(i) $E_{x}$ admits an ergodic, non-atomic, invarinat measure $\mu_{x}$.

(ii) If $x_{0} \neq x_{1}$ and $f: 2^{\mathbb{N}} \rightarrow 2^{\mathbb{N}}$ is a Borel function so that $y E_{x_{0}} z \Rightarrow f(y) E_{x_{1}} f(z)$, then there is a Borel $E_{x_{0}}$-invariant set $M$ of $\mu_{x_{0}}$-measure 1 such that $f(M)$ is contained in a single $E_{x_{1}}$-class. In particular, for any Borel set $N$ of $\mu_{x_{0}}$ measure $1, E_{x_{0}} \uparrow N \mathbb{Z}_{B} E_{x_{1}}$.

(iii) The assignment $x \mapsto E_{x}$ is Borel in the sense that $E \subseteq\left(2^{\mathbb{N}}\right)^{3}$ below is Borel:

$$
(x, y, z) \in E \Leftrightarrow y E_{x} z .
$$

Moreover, $x \mapsto \mu_{x}$ is Borel, where the Borel structure of the space of measures arises from the fact that each Borel probability measure on $2^{\mathbb{N}}$ is uniquely determined by a function $\varphi: 2^{<\mathbb{N}} \rightarrow[0,1]$ with $\varphi(\emptyset)=1$ and $\varphi(s)=\varphi\left(s^{\frown 0)+}\right.$ $\varphi\left(s^{\frown} 1\right)$ for all $s \in 2^{<\mathbb{N}}$.

Theorem 3. Every $\Sigma_{1}^{1}$ equivalence relation is Borel reducible to $\sim_{B}^{\text {ctbl }}$.

Proof. Without loss of generality, let $R$ be a $\Sigma_{1}^{1}$ equivalence relation on $2^{\mathbb{N}}$. By a result of Shelah (cf. 2], page 90) there is a standard Borel group $G$ and a Borel action of $G$ on $2^{\mathbb{N}}$ so that $R$ is the induced orbit equivalence relation. For each $x \in 2^{\mathbb{N}}$, define

$$
A_{x}=\left\{(y, g) \in 2^{\mathbb{N}} \times G \mid g \cdot y=x\right\} .
$$

Then $A_{x}$ is a Borel subset of $2^{\mathbb{N}} \times G$, hence is itself standard Borel. We then assign to each $x \in 2^{\mathbb{N}}$ the equivalence relation

$$
S_{x}=\bigsqcup_{(y, g) \in A_{x}} E_{y},
$$

where $\bigsqcup$ denotes the direct sum of equivalence relations and $E_{y}$ is as described in Theorem 9 above. More formally, $S_{x}$ is an equivalence relation on $A_{x} \times 2^{\mathbb{N}}$, and

$$
((y, g), z) S_{x}\left(\left(y^{\prime}, g^{\prime}\right), z^{\prime}\right) \Leftrightarrow y=y^{\prime} \text { and } g=g^{\prime} \text { and } z E_{y} z^{\prime} .
$$

Hence $S_{x}$ is Borel and each $S_{x}$-class is countable.

We check that, for $x, x^{\prime} \in 2^{\mathbb{N}}, x R x^{\prime}$ iff $S_{x} \sim_{B} S_{x^{\prime}}$. First suppose $x R x^{\prime}$. Then there is $h \in G$ such that $x^{\prime}=h \cdot x$. Define $f: A_{x} \times 2^{\mathbb{N}} \rightarrow A_{x^{\prime}} \times 2^{\mathbb{N}}$ by $f(y, g, z)=(y, h g, z)$ (we abbreviate $((y, g), z)$ by $(y, g, z))$. Then $f$ is Borel, and for $(y, g, z),\left(y^{\prime}, g^{\prime}, z^{\prime}\right) \in A_{x} \times 2^{\mathbb{N}}$,

$$
\begin{aligned}
(y, g, z) S_{x}\left(y^{\prime}, g^{\prime}, z^{\prime}\right) & \Leftrightarrow y=y^{\prime} \text { and } g=g^{\prime} \text { and } z E_{y} z^{\prime} \\
& \Leftrightarrow y=y^{\prime} \text { and } h g=h g^{\prime} \text { and } z E_{y} z^{\prime} \\
& \Leftrightarrow f(y, g, z) S_{x^{\prime}} f\left(y^{\prime}, g^{\prime}, z^{\prime}\right) .
\end{aligned}
$$

Therefore $S_{x} \leq_{B} S_{x^{\prime}}$. By symmetry, we have $S_{x} \sim_{B} S_{x^{\prime}}$.

Now suppose $x \mathbb{R} x^{\prime}$. Note that $E_{x} \leq_{B} S_{x}$, as witnessed by the map $z \mapsto\left(x, 1_{G}, z\right)$, where $1_{G}$ is the identity element of the group $G$. We just show that $E_{x} \mathbb{Z}_{B} S_{x^{\prime}}$, hence concluding that $S_{x} \mathbb{Z}_{B} S_{x^{\prime}}$ and $S_{x} \chi_{B} S_{x^{\prime}}$. Assume $f$ is a Borel reduction from $E_{x}$ into $S_{x^{\prime}}$, i.e., $f: 2^{\mathbb{N}} \rightarrow A_{x} \times 2^{\mathbb{N}}$ is such that $z E_{x} z^{\prime}$ iff $f(z) S_{x^{\prime}} f\left(z^{\prime}\right)$, for any $z, z^{\prime} \in 2^{\mathbb{N}}$. Letting $\pi_{1}(u, v, w)=(u, v)$, then we have in particular that, for any $z, z^{\prime} \in 2^{\mathbb{N}}$,

$$
z E_{x} z^{\prime} \Rightarrow \pi_{1}(f(z))=\pi_{1}\left(f\left(z^{\prime}\right)\right) .
$$


By ergodicity of $\mu_{x}$ given by Theorem 9 (i), there is a $\mu_{x}$-measure 1 set $M_{x}$ such that $\pi_{1}(f(z))$ is constant for $z \in M_{x}$. Say $\pi_{1}(f(z))=\left(y_{0}, g_{0}\right)$ for $z \in M_{x}$. Then $g_{0} \cdot y_{0}=x^{\prime}$, hence $y_{0} R x^{\prime}$ and in particular $y_{0} \neq x$. Letting $\pi_{2}(u, v, w)=w$, it follows that the map $z \mapsto \pi_{2}(f(z))$ is a reduction from $E_{x} \uparrow M_{x}$ into $E_{y_{0}}$. But since $y_{0} \neq x$, this contradicts Theorem 9 (ii).

Finally, by Theorem 9 (iii) it is easy to see that the map $x \mapsto S_{x}$ is Borel. Thus we have shown that there is a Borel reduction from $R$ into $\sim_{B}^{\text {ctbl }}$.

It is now fairly clear that the above proof establishes the following lemma.

Lemma 10. Let $A$ be a standard Borel space, $\gamma: A \rightarrow 2^{\mathbb{N}}$ a Borel function and $\Gamma$ the image of $\gamma$. Let $S$ be the equivalence relation defined by

$$
S=\bigsqcup_{a \in A} E_{\gamma(a)} .
$$

Let $x \notin \Gamma$. Then $E_{x} \not_{B} S$.

In fact, the above proof can be divided into two logical parts: one of them proves Lemma 10 from Theorem 9 and the other part finishes the argument by making use of the statement of Lemma 10, the Borelness (which is a moderate condition) provided by Theorem 9 (iii) and general facts about Borel equivalence relations. In the sequel when we prove analogous results about other classes of equivalence relations it suffices to verify the lemma and then we can repeat the rest of the proof verbatim (the Borelness is usually not a problem).

Adams and Kechris' original proof of Theorem 1 made use of a similar, in fact simpler, direct sum. Specifically, they assigned to each Borel set $A \subseteq 2^{\mathbb{N}}$ the equivalence relation

$$
E_{A}=\bigsqcup_{x \in A} E_{x} .
$$

Then by Lemma 10 and a similar argument as in the above proof, one can check that

$$
A_{1} \subseteq A_{2} \Leftrightarrow E_{A_{1}} \leq_{B} E_{A_{2}} .
$$

In the rest of this section we deal with $\sigma\left(\boldsymbol{\Sigma}_{1}^{1}\right)$-measurable reductions and prove Theorem 4. Following [1] we use the following notation. Given a Borel equivalence relation $E$ on a standard Borel space $X$, define $E^{*}$ or $\mathfrak{c} E$ to be the following direct sum

$$
\bigsqcup_{x \in \mathbb{N}^{\mathbb{N}}} E,
$$

i.e., the direct sum of continuum many copies of $E$. More formally, $E^{*}$ is an equivalence relation on $\mathbb{N}^{\mathbb{N}} \times X$, and

$$
(x, z) E^{*}\left(x^{\prime}, z^{\prime}\right) \Leftrightarrow x=x^{\prime} \text { and } z E z^{\prime} .
$$

Theorem 4. Every $\boldsymbol{\Sigma}_{1}^{1}$ or $\boldsymbol{\Pi}_{1}^{1}$ equivalence relation is Borel reducible to $\sim_{S}^{c t b l}$.

Proof. First let $R$ be a $\Sigma_{1}^{1}$ equivalence relation on $2^{\mathbb{N}}$. Let $C$ be a closed subset of $2^{\mathbb{N}}$ such that

$$
x R y \Leftrightarrow \exists z(x, y, z) \in C .
$$


Let $C_{x}=\left\{(y, z) \in 2^{\mathbb{N}} \times 2^{\mathbb{N}} \mid(x, y, z) \in C\right\}$. For each $x \in 2^{\mathbb{N}}$, define an equivalence relation $T_{x}$ by

$$
T_{x}=\bigsqcup_{(y, z) \in C_{x}} E_{y}^{*},
$$

where $E_{y}$ is again given by Theorem 9 . Then $T_{x}$ is a Borel equivalence relation on $C_{x} \times \mathbb{N}^{\mathbb{N}} \times 2^{\mathbb{N}}$ and the map $x \mapsto T_{x}$ is clearly Borel.

We check that, for $x, x^{\prime} \in 2^{\mathbb{N}}, x R x^{\prime}$ iff $T_{x} \sim_{S} T_{x^{\prime}}$. First suppose $x R x^{\prime}$. Consider the set

$$
C_{x, x^{\prime}}=\left\{\left(y, z, z^{\prime}\right) \mid(y, z) \in C_{x} \text { and }\left(y, z^{\prime}\right) \in C_{x^{\prime}}\right\} .
$$

$C_{x, x^{\prime}}$ is a Borel subset of the product space $C_{x} \times 2^{\mathbb{N}}$ and moreover, for any $(y, z) \in$ $C_{x}$, there is $z^{\prime} \in 2^{\mathbb{N}}$ such that $\left(y, z, z^{\prime}\right) \in C_{x, x^{\prime}}$. By the Jankov-von Neumann Uniformization Theorem ([9], Theorem 18.1), there is a $\sigma\left(\boldsymbol{\Sigma}_{1}^{1}\right)$-measurable uniformization function $g: C_{x} \rightarrow 2^{\mathbb{N}}$ such that $(y, g(y, z)) \in C_{x^{\prime}}$ for any $(y, z) \in C_{x}$. We then define, for $((y, z), u, v) \in C_{x} \times \mathbb{N}^{\mathbb{N}} \times 2^{\mathbb{N}}$,

$$
f(y, z, u, v)=(y, g(y, z),\langle u, z\rangle, v)
$$

where we abbreviate again $((y, z), u, v)$ by $(y, z, u, v)$ and $\langle\cdot, \cdot\rangle$ is a fixed Borel bijection from $\mathbb{N}^{\mathbb{N}} \times 2^{\mathbb{N}}$ onto $\mathbb{N}^{\mathbb{N}}$. Then $f$ is a $\sigma\left(\boldsymbol{\Sigma}_{1}^{1}\right)$-measurable function such that, for $(y, z, u, v),\left(y^{\prime}, z^{\prime}, u^{\prime}, v^{\prime}\right) \in C_{x} \times \mathbb{N}^{\mathbb{N}} \times 2^{\mathbb{N}}$,

$$
\begin{aligned}
(y, z, u, v) T_{x}\left(y^{\prime}, z^{\prime}, u^{\prime}, v^{\prime}\right) & \Leftrightarrow y=y^{\prime}, z=z^{\prime}, u=u^{\prime} \text { and } v E_{y} v^{\prime} \\
& \Leftrightarrow y=y^{\prime},\langle u, z\rangle=\left\langle u^{\prime}, z^{\prime}\right\rangle \text { and } v E_{y} v^{\prime} \\
& \Leftrightarrow y=y^{\prime}, g(y, z)=g\left(y^{\prime}, z^{\prime}\right),\langle u, z\rangle=\left\langle u^{\prime}, z^{\prime}\right\rangle \text { and } v E_{y} v^{\prime} \\
& \Leftrightarrow f(y, z, u, v) T_{x^{\prime}} f\left(y^{\prime}, z^{\prime}, u^{\prime}, z^{\prime}\right) .
\end{aligned}
$$

This shows that $T_{x} \leq_{S} T_{x^{\prime}}$. By symmetry, we have $T_{x} \sim_{S} T_{x^{\prime}}$.

Now suppose $x \not R x x^{\prime}$. Then again $E_{x} \leq_{B} T_{x}$, therefore it suffices to check that $E_{x} \leq_{S} T_{x^{\prime}}$. Assume $E_{x} \leq_{S} T_{x^{\prime}}$ via function $f$. Then there is a $\mu_{x}$-measure 1 set $M_{x}$ such that $f$ is Borel on $M_{x}$, thus $E_{x}\left\lceil M_{x} \leq_{B} T_{x^{\prime}}\right.$ via $f$. We can then argue as in the proof of Theorem 3 to conclude that there must be some $y$ for which $E_{y}$ appears as a summand of $T_{x^{\prime}}$ so that $E_{x} \uparrow N_{x} \leq_{B} E_{y}$ for some $\mu_{x}$-measure 1 set $N_{x} \subseteq M_{x}$. But $x \neq y$ since $y R x^{\prime}$, and we arrive at a contradiction to Theorem 9 (ii) again.

This finishes the proof for $\boldsymbol{\Sigma}_{1}^{1}$ equivalence relations.

Now let $R$ be a $\Pi_{1}^{1}$ equivalence relation on $2^{\mathbb{N}}$ and let $F$ be a Borel subset of $\left(2^{\mathbb{N}}\right)^{3}$ such that

$$
x R y \Leftrightarrow \forall z(x, y, z) \in F .
$$

For each $x \in 2^{\mathbb{N}}$, define an equivalence relation $U_{x}$ by

$$
U_{x}=\bigsqcup_{(x, y, z) \notin F} E_{y}^{*} .
$$

Then $E_{y}$ appears in the direct sum of $U_{x}$ iff $y \not R x$. If $x R x^{\prime}$, then as before we have $U_{x} \sim_{S} U_{x^{\prime}}$. If $x \not R x^{\prime}$, then $E_{x^{\prime}} \leq_{B} U_{x}$ but $E_{x^{\prime}} \not_{S} U_{x^{\prime}}$, so $U_{x} \chi_{S} U_{x^{\prime}}$.

Note that in the above proof we relied on a stronger claim that $E_{x} \not_{S} S$ when $E_{x}$ does not appear as a summand of the direct sum $S$. The rest of the argument again only used general facts about Borel equivalence relations. Also note that the $\boldsymbol{\Sigma}_{1}^{1}$ part of the proof would work fine if we used direct sums as those in the proof of Theorem 3. The current proof makes the $\boldsymbol{\Pi}_{1}^{1}$ part much easier. 


\section{Essentially uncountable EQUivalence RELATions}

Throughout this section we fix $\left\{E_{x}\right\}_{x \in 2^{\mathbb{N}}}$ to be the system of countable Borel equivalence relations given by Theorem 9 of the last section. In this section we consider the system $\left\{E_{x}^{\omega}\right\}_{x \in 2^{\mathbb{N}}}$ and prove that all the previous results about countable Borel equivalence relations have counterparts in the interval $\left[E_{0}^{\omega}, E_{\infty}^{\omega}\right]$.

Note that the Borelness of the enumeration of the system follows from Theorem 9 (iii), therefore it is indeed enough to verify the stronger version of Lemma 10 for this new system.

Lemma 11. Let $A$ be a standard Borel space, $\gamma: A \rightarrow 2^{\mathbb{N}}$ a Borel function and $\Gamma$ the image of $\gamma$. Let $S$ be the equivalence relation defined by

$$
S=\bigsqcup_{a \in A} E_{\gamma(a)}^{\omega} .
$$

Let $x \notin \Gamma$. Then $E_{x}^{\omega} \not E_{S} S$.

Proof. We actually show that $E_{x} \mathbb{Z}_{S} S$. Since $E_{x} \leq_{B} E_{x}^{\omega}$, this implies that $E_{x}^{\omega} \mathbb{E}_{S}$ $S$.

Assume $f$ is a $\sigma\left(\boldsymbol{\Sigma}_{1}^{1}\right)$-measurable reduction from $E_{x}$ into $S$, i.e., $f: 2^{\mathbb{N}} \rightarrow$ $A \times\left(2^{\mathbb{N}}\right)^{\omega}$ is $\sigma\left(\boldsymbol{\Sigma}_{1}^{1}\right)$-measurable so that $y E_{x} z \Leftrightarrow f(y) S f(z)$ for any $y, z \in 2^{\mathbb{N}}$. Let $g, f_{0}, f_{1}, \ldots$ be an infinite sequence of $\sigma\left(\boldsymbol{\Sigma}_{1}^{1}\right)$-measurable functions such that, for any $y \in 2^{\mathbb{N}}$

$$
f(y)=\left(g(y), f_{0}(y), f_{1}(y), \cdots\right) \in A \times\left(2^{\mathbb{N}}\right)^{\omega} .
$$

Then by the definition of $S$, we have

$$
y E_{x} z \Leftrightarrow g(y)=g(z) \text { and } f_{i}(y) E_{\gamma(g(y))} f_{i}(z) \text { for all } i \in \omega .
$$

Now by Theorem 9 , there are subsets $M, N_{0}, N_{1}, \ldots$ of $2^{\mathbb{N}}$ each of $\mu_{x}$-measure 1 , such that $g$ is constant on $M$, say with value $u_{0}$, and for each $i, f_{i} \uparrow N_{i}$ is Borel and $f_{i}\left(N_{i}\right)$ is contained in a single $E_{\gamma\left(u_{0}\right)}$-class. Put

$$
W=M \cap \bigcap_{i \in \omega} N_{i}
$$

Then $W$ is of $\mu_{x}$-measure $1, f \uparrow W$ is Borel and $f(W)$ is contained in a single $S$-class. It follows that $W$ is contained in a single $E_{x}$-class, contradicting non-atomicity of $\mu_{x}$

A slight modification of the above proof also establishes the following stronger result, which will be useful in our subsequent proofs.

Lemma 12. Let $A$ be a standard Borel space, $\gamma: A \rightarrow 2^{\mathbb{N}}$ a Borel function and $\Gamma$ the image of $\gamma$. Let $S$ be the equivalence relation defined by

$$
S=\bigsqcup_{a \in A} E_{\gamma(a)}^{\omega} .
$$

Let $x \notin \Gamma$. Then $E_{x} \uparrow M \not \mathbb{S}_{S} S$ for any $\mu_{x}$-measure 1 set $M$. 
We next check that the direct sums are in fact within the interval $\left[E_{0}^{\omega}, E_{\infty}^{\omega}\right]$.

Lemma 13. Let $A$ be a standard Borel space and $\gamma: A \rightarrow 2^{\mathbb{N}}$ a Borel function. Let $S$ be the equivalence relation defined by

$$
S=\bigsqcup_{a \in A} E_{\gamma(a)}^{\omega} .
$$

Then $E_{0}^{\omega} \leq_{B} S \leq_{B} E_{\infty}^{\omega}$.

Proof. It is clear that $E_{0}^{\omega} \leq_{B} S$. To see that $S \leq_{B} E_{\infty}^{\omega}$, we consider

$$
T=\bigsqcup_{a \in A} E_{\gamma(a)} \text {. }
$$

It is clear that $T$ is a countable Borel equivalence relation, therefore $T \leq_{B} E_{\infty}$. We claim that $S \leq_{B} T^{\omega}$, which suffices since $T^{\omega} \leq_{B} E_{\infty}^{\omega}$.

For $\left(a, x_{0}, x_{1}, \cdots\right) \in A \times\left(2^{\mathbb{N}}\right)^{\omega}$, define

$$
f\left(a, x_{0}, x_{1}, \cdots\right)=\left(\left(a, x_{0}\right),\left(a, x_{1}\right), \cdots\right) \in\left(A \times 2^{\mathbb{N}}\right)^{\omega} .
$$

Then

$$
\begin{aligned}
\left(a, x_{0}, x_{1}, \cdots\right) S\left(b, y_{0}, y_{1}, \cdots\right) & \Leftrightarrow a=b \text { and for any } i \in \omega, x_{i} E_{\gamma(a)} y_{i} \\
& \Leftrightarrow \text { for any } i \in \omega, a=b \text { and } x_{i} E_{\gamma(a)} y_{i} \\
& \Leftrightarrow \quad \text { for any } i \in \omega,\left(a, x_{i}\right) T\left(b, y_{i}\right) \\
& \Leftrightarrow\left(\left(a, x_{0}\right),\left(a, x_{1}\right), \cdots\right) T^{\omega}\left(\left(b, y_{0}\right),\left(b, y_{1}\right), \cdots\right) .
\end{aligned}
$$

This finishes the proof of the lemma.

We now have the following immediate corollaries.

Theorem 5. The structure of Borel sets with inclusion can be embedded into $\left(\left[E_{0}^{\omega}, E_{\infty}^{\omega}\right], \leq_{B}\right)$.

Theorem 6 (First half). The following facts hold for the interval $\mathcal{I}=\left[E_{0}^{\omega}, E_{\infty}^{\omega}\right]$ :

(a) Every $\boldsymbol{\Sigma}_{1}^{1}$ equivalence relation is Borel reducible to $\sim_{B}^{\mathcal{I}}$.

(b) Every $\boldsymbol{\Sigma}_{1}^{1}$ or $\boldsymbol{\Pi}_{1}^{1}$ equivalence relation is Borel reducible to $\sim_{S}^{\mathcal{I}}$.

The clauses (c)-(e) of Theorem 6 can be proved verbatim from the AdamsKechris' proofs in [1] for the corresponding statements for countable Borel equivalence relations. For convenience of the reader we sketch the proofs below.

Theorem 6 (Second half). The following facts hold for the interval $\mathcal{I}=\left[E_{0}^{\omega}, E_{\infty}^{\omega}\right]$ :

(c) $\sim_{B}^{\mathcal{I}}$ is $\boldsymbol{\Sigma}_{2}^{1}$-complete.

(d) $\sim_{S}^{\mathcal{I}}$ is $\boldsymbol{\Pi}_{2}^{1}$-hard.

(e) There are $E, F \in \mathcal{I}$ such that $E \leq_{S} F$ but $E \not \leq_{B} F$.

Proof. First by modifying Theorem 9 in an obvious way we can think of all $E_{x}$ to be equivalence relations on $\mathbb{N}^{\mathbb{N}}$ instead of on $2^{\mathbb{N}}$. Also we think of the index set to be $\mathbb{N}^{\mathbb{N}}$ instead of $2^{\mathbb{N}}$. It is clear that (e) follows from (c) and (d). To prove (c) and (d), consider trees on $\mathbb{N} \times \mathbb{N}$. For a tree $T$ on $\mathbb{N} \times \mathbb{N}$, let $[T]$ denote the set of infinite branches of $T$. Then $[T]$ is a closed subset of $\left(\mathbb{N}^{\mathbb{N}}\right)^{2}$. Associate with $T$ the equivalence relation

$$
E_{T}=\bigsqcup_{(x, y) \in[T]}\left(E_{x}^{\omega}\right)^{*}
$$


and let

$$
E=\bigsqcup_{x \in \mathbb{N}^{\mathbb{N}}}\left(E_{x}^{\omega}\right)^{*}
$$

For (c) we shall show that the class $\left\{R \in \mathcal{I} \mid R \sim_{B} E\right\}$ is $\boldsymbol{\Sigma}_{2}^{1}$-complete. This is done in turn by considering the set FBU of trees $T$ on $\mathbb{N} \times \mathbb{N}$ where $T$ has a full Borel uniformization, i.e., there is a Borel function $u: \mathbb{N}^{\mathbb{N}} \rightarrow \mathbb{N}^{\mathbb{N}}$ such that, for every $x \in \mathbb{N}^{\mathbb{N}},(x, u(x)) \in[T]$. It is a result of Steel and there is a proof in [1] that FBU is $\Sigma_{2}^{1}$-complete. (c) would be established by verifying that $T \in \mathrm{FBU}$ iff $E \sim_{B} E_{T}$.

Suppose that $u$ is a full Borel uniformization of $[T]$. Then a reduction from $E$ into $E_{T}$ can clearly be defined by $f: \mathbb{N}^{\mathbb{N}} \times \mathbb{N}^{\mathbb{N}} \times\left(\mathbb{N}^{\mathbb{N}}\right)^{\omega} \rightarrow[T] \times \mathbb{N}^{\mathbb{N}} \times\left(\mathbb{N}^{\mathbb{N}}\right)^{\omega}$ where

$$
f(x, y, \vec{z})=(x, u(x), y, \vec{z}) .
$$

Since $u$ is Borel, so is $f$, and hence $E \leq_{B} E_{T}$. On the other hand,

$$
E_{T} \leq_{B} \bigsqcup_{(x, y) \in\left(\mathbb{N}^{N}\right)^{2}}\left(E_{x}^{\omega}\right)^{*} \leq_{B} \bigsqcup_{x \in \mathbb{N}^{\mathbb{N}}} \mathfrak{c}\left(\mathfrak{c} E_{x}^{\omega}\right) \cong_{B} E
$$

where $\cong_{B}$ denotes Borel isomorphism. Therefore $E_{T} \leq_{B} E$ and hence $E_{T} \sim_{B} E$.

Conversely, suppose $E \sim_{B} E_{T}$. In particular, there is a Borel function $g$ : $\mathbb{N}^{\mathbb{N}} \times \mathbb{N}^{\mathbb{N}} \times\left(\mathbb{N}^{\mathbb{N}}\right)^{\omega} \rightarrow[T] \times \mathbb{N}^{\mathbb{N}} \times\left(\mathbb{N}^{\mathbb{N}}\right)^{\omega}$ reducing $E$ into $E_{T}$. For each $x \in \mathbb{N}^{\mathbb{N}}$, the function $g_{x}(y, \vec{z})=g(x, y, \vec{z})$ is a Borel reduction of $\left(E_{x}^{\omega}\right)^{*}$ into $E_{T}$. Fix a Borel function $\rho$ reducing each $E_{x}$ into $\left(E_{x}^{\omega}\right)^{*}$ (so that $\rho$ does not depend on $x$ ). Then $g_{x} \circ \rho$ is a Borel reduction of $E_{x}$ into $E_{T}$, thus in particular,

$$
y E_{x} z \rightarrow \pi_{1}\left(\left(g_{x} \circ \rho\right)(y)\right)=\pi_{1}\left(\left(g_{x} \circ \rho\right)(z)\right) \in[T]
$$

where $\pi_{1}(u, v, y, \vec{z})=(u, v)$. By ergodicity of $\mu_{x}$, there is a set $M_{x}$ of $\mu_{x}$-measure 1 so that $\pi_{1} \circ g_{x} \circ \rho$ is constant on $M_{x}$, say with value $(u(x), f(x)) \in[T]$. Then $\pi_{2} \circ g_{x} \circ \rho$ is a Borel reduction of $E_{x} \uparrow M_{x}$ into $\left(E_{u(x)}^{\omega}\right)^{*}$, where $\pi_{2}(u, v, y, \vec{z})=(y, \vec{z})$. Now by Lemma 12, we must have $u(x)=x$. Thus $f$ is a full uniformization of $[T]$. $f$ is Borel since its graph $G_{f}$ is given by the computation

$$
(x, y) \in G_{f} \Leftrightarrow y=f(x) \Leftrightarrow \mu_{x}\left(\left\{z \mid\left(\pi_{1} \circ g_{x} \circ \rho\right)(z)=(x, y)\right\}\right)=1
$$

and this is Borel by [9, Theorem 17.25.

Now for the proof of (d) we consider the set FP of trees $T$ on $\mathbb{N} \times \mathbb{N}$ where $[T]$ has full projection, i.e., for every $x \in \mathbb{N}^{\mathbb{N}}$ there is some $y \in \mathbb{N}^{\mathbb{N}}$ with $(x, y) \in[T]$. By the Jankov-von Neumann Uniformization Theorem again, $T \in \mathrm{FP}$ iff $[T]$ admits a full $\sigma\left(\boldsymbol{\Sigma}_{1}^{1}\right)$-measurable uniformization. And it is well known that FP is $\boldsymbol{\Pi}_{2}^{1}$-hard. Note that a $\sigma\left(\boldsymbol{\Sigma}_{1}^{1}\right)$-measurable function is Borel on a measure 1 set. An obvious modification of the above argument for part (c) shows that $T \in \mathrm{FP}$ iff $E \sim_{S} E_{T}$, therefore showing that the set $\left\{R \mid R \sim_{S} E\right\}$ is $\Pi_{2}^{1}$-hard.

In this proof one needs to apply one more round of ergodicity and Lemma 12 other than the generalities of Borel equivalence relations. Fortunately, we shall see in the other examples to come that there are natural analogs of the ergodicity condition, sometimes in the form of generic ergodicity, and that of Lemma 12. 


\section{Another Class of Borel equivalence Relations}

The system of equivalence relations which serves our purposes in this section are the so-called Tsirelson equivalence relations. The notion of Tsirelson ideals was first studied by Farah ([5], 6]) and Velickovic ([11), with the fundamental ideas borrowed from the analysis of Tsirelson spaces by functional analysts. The Tsirelson ideals are Borel ideals on $\mathcal{P}(\omega)$ and hence naturally induces Borel equivalence relations on $2^{\mathbb{N}}$. It will not be important how the equivalence relations are actually defined. Let us only mention here the basic properties relevant to our application below. The details of the proofs can be found in [6] and [7].

Theorem 14 (Farah-Hjorth). There is a system of Borel equivalence relations $\left\{E_{\alpha}\right\}_{\alpha \in(0,1)}$, where $(0,1)$ is the open interval of real numbers between 0 and 1 , which has the following properties:

(i) For each $\alpha \in(0,1), E_{\alpha}$ is a Borel equivalence relation on $2^{\mathbb{N}}$ so that every $E_{\alpha}$-class is meager and $E_{\alpha}$ is generically $F_{2}$-ergodic, i.e., if $f: 2^{\mathbb{N}} \rightarrow\left[2^{\mathbb{N}}\right]^{\omega}$ is a Borel function such that $x E_{\alpha} y \Rightarrow f(x)=f(y)$, then $f$ is constant on a Borel comeager set.

(ii) If $\alpha \neq \beta$ are both reals in $(0,1)$, and $F$ is a Borel equivalence relation such that $F \leq_{B} E_{\alpha}$ and $F \leq_{B} E_{\beta}$, then $F$ is essentially countable.

(iii) The assignment $\alpha \mapsto E_{\alpha}$ is Borel.

In the clause (i) above a much stronger statement is true by Hjorth's theory of turbulence (7]): every $E_{\alpha}$ is generically $S_{\infty}$-ergodic. Roughly speaking, each $E_{\alpha}$ is not Borel reducible to the isomorphism of countable models in a very strong sense: if there were such a reduction, then there would be a comeager $E_{\alpha}$-class, which is not the case. It is then clear that any direct sum of the Tsirelson relations $E_{\alpha}$ is not Borel reducible to the isomorphism of countable models, in other words, they all belong to $\mathcal{E}^{\text {turb }}$.

Let us first prove an analog of Lemma 10 to illustrate the specialty of the argument.

Lemma 15. Let $A$ be a standard Borel space, $\gamma: A \rightarrow(0,1)$ a Borel function and $\Gamma$ the image of $\gamma$. Let $S$ be the equivalence relation defined by

$$
S=\bigsqcup_{a \in A} E_{\gamma(a)} .
$$

Let $\alpha \notin \Gamma$. Then $E_{\alpha} \not_{B} S$.

Proof. Assume that $f$ is a Borel reduction from $E_{\alpha}$ into $S$, i.e., $f: 2^{\mathbb{N}} \rightarrow A \times 2^{\mathbb{N}}$ is such that $x E_{\alpha} y$ iff $f(x) S f(y)$. Letting $\pi_{1}(u, v)=u$ and $\pi_{2}(u, v)=v$, we have that, for $x, y \in 2^{\mathbb{N}}$,

$$
x E_{\alpha} y \Rightarrow \pi_{1}(f(x))=\pi_{1}(f(x)) .
$$

Fix a Borel embedding $\rho$ from $A$ into $2^{\mathbb{N}}$. By the generic ergodicity given by Theorem $14(\mathrm{i})$, there is a Borel comeager subset $C$ of $2^{\mathbb{N}}$ such that $\pi_{1} \circ f$ is constant on $C$, say with value $a$. Let $\beta=\gamma(a)$. It then follows that $\pi_{2} \circ f$ is a Borel reduction from $E_{\alpha} \uparrow C$ into $E_{\beta}$. Moreover, $\beta \neq \alpha$.

Denote $F=E_{\alpha}\left\lceil C\right.$. Then $F \leq_{B} E_{\alpha}$ via the identity function and $F \leq_{B} E_{\beta}$ via $\pi_{2} \circ f$. By Theorem 14 (ii), we conclude that $F$ is essentially countable. In particular, there is a Borel function $g: C \rightarrow\left[2^{\mathbb{N}}\right]^{\omega}$ such that, for any $x, y \in C, x F y$ iff $g(x)=g(y)$. We extend $g$ to $g^{\prime}$ defined on the whole of $2^{\mathbb{N}}$ by letting $g^{\prime}$ be some 
constant on $2^{\mathbb{N}} \backslash C$. Then $g^{\prime}: 2^{\mathbb{N}} \rightarrow\left[2^{\mathbb{N}}\right]^{\omega}$ is a Borel function such that, for any $x, y \in 2^{\mathbb{N}}$,

$$
x E_{\alpha} y \Rightarrow g^{\prime}(x)=g^{\prime}(y) .
$$

By the generic ergodicity again, there is a comeager set $D \subseteq 2^{\mathbb{N}}$ such that $g^{\prime}$ is constant on $D$. Now $C \cap D$ is comeager and it follows that $C \cap D$ is contained in a single $E_{\alpha}$-class. But this is absurd since every $E_{\alpha}$-class is meager.

The analog of Lemma 12 can now be stated with the measure 1 set replaced by a comeager set, whose proof is similar to the above.

Lemma 16. Let $A$ be a standard Borel space, $\gamma: A \rightarrow(0,1)$ a Borel function and $\Gamma$ the image of $\gamma$. Let $S$ be the equivalence relation defined by

$$
S=\bigsqcup_{a \in A} E_{\gamma(a)} .
$$

Let $\alpha \notin \Gamma$. Then $E_{\alpha}\left\lceil C \not \mathbb{S}_{S} S\right.$ for any comeager set $C$.

By now it is clear that the Adams-Kechris' results have counterparts in $\mathcal{E}^{\text {turb }}$.

Theorem 7. The following facts hold for $\mathcal{E}^{\text {turb }}$ :

(a) The structure of Borel sets with inclusion can be embedded into $\left(\mathcal{E}^{\text {turb }}, \leq_{B}\right)$.

(b) Every $\boldsymbol{\Sigma}_{1}^{1}$ equivalence relation is Borel reducible to $\sim_{B}^{\text {turb }}$.

(c) Every $\boldsymbol{\Sigma}_{1}^{1}$ or $\boldsymbol{\Pi}_{1}^{1}$ equivalence relation is Borel reducible to $\sim_{S}^{\text {turb }}$.

(d) $\sim_{B}^{\text {turb }}$ is $\boldsymbol{\Sigma}_{2}^{1}$-complete.

(e) $\sim_{S}^{\text {turb }}$ is $\Pi_{2}^{1}$-hard.

(f) There are $E, F \in \mathcal{E}^{\text {turb }}$ such that $E \leq_{S} F$ but $E \not_{B} F$.

Proof. (a) and (b) are immediate from Lemma 15. (c) is immediate from Lemma 16. For (d) repeat the proof of Theorem 6 (c), where we replace the ergodicity of the measures by the generic ergodicity and use Lemma 16. For (e) note in extra that every $\sigma\left(\boldsymbol{\Sigma}_{1}^{1}\right)$-measurable function is Baire measurable, hence is Borel on a Borel comeager set. Finally (f) follows from (d) and (e).

\section{Borel AUTOMORPHISMS AND FURTHER QUESTIONS}

Inspired by the work [4] of Eigen-Hajian-Weiss, Clemens ([3]) considered the structure of Borel automorphisms with Borel embedding or Borel isomorphism. Even if these are not equivalence relations, it still makes sense to talk about direct sums of Borel automorphisms. In [3] Clemens essentially proved the analog of Theorem 9 or 14 for Borel automorphisms and hence was able to recover all the Adams-Kechris' results for this class of objects. Here let us just remark that our proofs of Theorems 3 and 4 can be repeated verbatim to give the following stronger result.

Theorem 8. Every $\boldsymbol{\Sigma}_{1}^{1}$ equivalence relation is Borel reducible to the Borel isomorphism of Borel automorphisms. Every $\boldsymbol{\Sigma}_{1}^{1}$ or $\boldsymbol{\Pi}_{1}^{1}$ equivalence relation is Borel reducible to the $\sigma\left(\boldsymbol{\Sigma}_{1}^{1}\right)$-measurable isomorphism of Borel automorphisms.

We close by mentioning some further questions.

In [1] the authors asked whether every $\Pi_{1}^{1}$ equivalence relation is Borel reducible to $\sim_{B}^{\text {ctbl }}$. This still seems open, together with the obvious modifications for other classes of objects we discussed in this paper. 
For the equivalence relations constructed in section 4 (direct sums of the Tsirelson equivalence relations), we do not know whether they are induced by Polish group actions or Borel reducible to such. This would be the case if all of them are Borel reducible to a fixed Borel equivalence relation induced by a Polish group action, as given by the argument of Lemma 13 .

Finally, we do not know if the Adams-Kechris technique can be applied to nonBorel equivalence relations, especially to orbit equivalence relations of Polish group actions.

\section{REFERENCES}

[1] S. Adams And A. S. Kechris, Linear algebraic groups and countable Borel equivalence relations, J. Amer. Math. Soc. 13 (2000), 909-943. CMP 2000:16

[2] H. Becker And A. S. Kechris, The descriptive set theory of Polish group actions, London Mathematical Society Lecture Notes Series 232, Cambridge University Press, 1996. MR 98d:54068

[3] J. D. Clemens, Borel automorphisms, handwritten notes, 1999.

[4] S. Eigen, A. Hajian, And B. Weiss, Borel automorphisms with no finite invariant measure, Proc. Amer. Math. Soc. 126 (1998), no. 12, 3619-3623. MR 99b:28024

[5] I. FARAh, Ideals induced by Tsirelson submeasures, Fund. Math. 159 (1999), no. 3, 243-258. CMP 99:11

[6] I. FARAH, Basis problem for turbulent actions I: Tsirelson submeasures, Annals of Pure and Applied Logic, to appear.

[7] G. HJoRTh, Classification and orbit equivalence relations, Mathematical Surveys and Monographs, vol. 75, American Mathematical Society, 2000. MR 2000k:03097

[8] G. HJorth And A. S. Kechris, The complexity of the classification of Riemann surfaces and complex manifolds, Illinois J. Math. 44 (2000), no. 1, 104-137. MR 2000m:03115

[9] A. S. Kechris, Classical descriptive set theory, Springer-Verlag, New York, 1995 . MR 96e:03057

[10] A. Louveau and B. Velickovic, A note on Borel equivalence relations, Proc. Amer. Math. Soc. 120 (1994), no. 1, 255-259. MR 94f:54076

[11] B. Velickovic, A note on Tsirelson type ideals, Fund. Math. 159 (1999), no. 3, 259-268. MR 2000f:03142

Department of Mathematics, California Institute of Technology, Pasadena, CaliFORNIA 91125

E-mail address: sugao@its.caltech.edu 